\title{
La Chilomastiase
}

II serait abusif de prétendre que les parasitoses digestives subissent la loi d'une mystérieuse mode; néanmoins il est frappant, à la lecture régulière des périodiques, specialises ou non, de voir se multiplier les observations d'infestation par des agents reputes exceptionnels, n'évo-quant souvent que des souvenirs propédeutiques. II en a été ainsi par exemple de la grande douve du foie dont nombre de cas furent relates en France ces dernières années.

Ces reflexions nous sont suggérées par un recent mémoire italien sur Yínfestatíon par Chilomastíx Mesnílí (La Chílomastíasí par G. Mastrandrea et M. Mazzettí, Rass. ital. gastroenter. 4: 668-705, dec. 1958), où l'on apprend que cette parasitose n'est pas aussi rare que classiquement admis dans les traités. Sa distribution géographi-que est extrêmement étendue; elle intéresse surtout la zone tropicale, l'Afrique du nord, les Etats-Unis et, en Europe, la Yougoslavie. Les auteurs en ont observe quatre cas en Italie.

Chilomastix mesnili est exclusivement parasite de Thomme; il fut décrit en 1854 par Davaíne dans des selles de cholériques, sous le nom de Cercomonas Hominis. II fut ensuite redécouvert et rebaptisé à maintes reprises, fréquemment confondu avec Trichomonas intesti-nalis et avec Tagent de la lambliase, Giardia intestinalis, avec lesquels il présente quelques analogies. Dans sa forme vegetative, le proto-zoaire est un tétra-flagellé piriforme, de 13-24 $\mu / 5-6 \mu$ il a 6-9 $\mu$ /77,5 à l'état kystique. Le contage est essentiellement inter-humain, la transmission des kystes se fait par les aliments et $\Gamma$ eau; la mouche domestique peut servir de véhicule. La recherche coprologique paraît assez delicate et nécessite quelques precautions: après purgation, Гéchantillon sera prélevé non pas sur la premiere selle émise, mais bien sur les suivantes, car le parasite habite surtout le caecum. Quant aux techniques de coloration et de culture, nous renvoyons le lec-teur à la monographie originale, où elles sont exposées dans tout le detail souhaitable.

Le pouvoir pathogène des Chilomastix a été fort discuté. Et il l'est encore. Gertes, il est vraisemblable que le protozoaire soit fréquem-

Editorial

411

ment un banal saprophyte, ou encore un germe d'accompagnement dont la pullulation est favorisée par une côlite d'autre origine (syndrome entéro-rénal de Heitz-Boyer, amibiase). Toutefois, sa patho-génicité propre ne saurait être niée: il existe bien, en effet, une «Chilomastiase», caractérisée par un syndrome colitique plus ou moins aigu, dysentériforme, accompagné de phénomènes généraux tels qu'inappétence, nausées, coliques; la diarrhée peut aussi être isolée, avec conservation d'un bon état general. La réalité de ce syndrome colitique, qui d'ailleurs ne possède aucun caractère spécifique, est démontrée par les nombreuses observations où la guérison ne survient qu'après disparition de Гagent causal, et ce résultat n'est obtenu que par une thérapeutique qui, elle, est spécifique. Ajoutons que la coprologie montre des signes d'indigestion côlique. L'éosino-philie sanguine semble être modérée, encore que peu fréquente. 
Le traitement est identique à celui de la lambliase: derives de Tacridine (Atébrine, Acranil, à la dose de $0,30 \mathrm{~g}$. par jour durant 7 jours; cure à répéter après interruption de 20 jours), qui sont rapide-ment efficaces.

La monographie dont nous venons de faire une trop bref resume est très complete; les auteurs y font un historique détaillé et plein d'intérêt, Гétude zoologique est faite avec minutie, de même que $\Gamma$ exposé du tableau clinique et des techniques de recherche parasito-logique. L'abondante bibliographie témoigne d'un louable souci de documentation. Ce travail a le mérite de constituer une mise au point sur un sujet qui, sans être neuf, a Гintérêt de prêter encore à contro-verse. Les traités de gastro-entérologie ne lui consacrent que quelques lignes et minimisent son importance. II apparait souvent que Ton n'accorde pas aux parasitoses intesti-nales toute $\Gamma$ attention souhaitable, hormis bien entendu celles dont le diagnostic s'impose à l'ceil nu. Le spécialiste se voit en effet fré-quemment consulté pour une diarrhée au long cours, sur laquelle des mois durant de banales medications désinfectantes n'ont aucune prise, et qui cede en quelques jours à une thérapeutique causale: nous pensons en particulier à la lambliase (dont la découverte fréquente par tubage duodenal a fait si couramment accréditer la notion d'un habitat biliaire). II importe de préciser que le médecin n'est pas toujours blamable de pareilles errances: une réponse negative après examen coprologique hâtif, ou fait par un laboratoire peu competent en parasitologie, peut l'induire en confusion. Mais il ne devra en 